\title{
Thermal Conductivity of a Monolayer of Exfoliated Graphite Nanoplatelets Prepared by Liquid-Liquid Interfacial Self-Assembly
}

\author{
Jinglei Xiang and Lawrence T. Drzal \\ Composite Materials and Structures Center, Department of Chemical Engineering and Materials Science, \\ Michigan State University, East Lansing, MI 48824-1226, USA \\ Correspondence should be addressed to Lawrence T. Drzal, drzal@egr.msu.edu
}

Received 26 August 2010; Accepted 31 October 2010

Academic Editor: P. Panine

Copyright ( $) 2010$ J. Xiang and L. T. Drzal. This is an open access article distributed under the Creative Commons Attribution License, which permits unrestricted use, distribution, and reproduction in any medium, provided the original work is properly cited.

A monolayer film composed of exfoliated graphite nanoplatelets (xGnPs) was extracted from a chloroform-water interface and supported on a glass substrate. The nanoplatelets are interconnected at the edges without overlapping forming a very densely packed structure with uniform thickness. Micro-Raman spectroscopy with a $50 \mathrm{~mW} 532 \mathrm{~nm}$ laser generating heat at the center of a $\mathrm{xGnP}$ sample was used to probe the thermal conductivity of the $\mathrm{xGnP}$ monolayer at different power levels. The Raman $\mathrm{G}$ peak shift of graphite was used to record the local temperature rise in the monolayer. The cross-sectional area of heat conduction is determined by the thickness of individual nanoplatelets. A UV-Vis spectrometer was used to measure the absorption of light by the monolayer. Depending on the interface density, the thermal conductivities are around $380 \mathrm{~W} / \mathrm{m} \mathrm{K}$ and $290 \mathrm{~W} / \mathrm{m} \mathrm{K}$ for monolayers with average particle size of $10 \mu \mathrm{m}$ and $5 \mu \mathrm{m}$, respectively.

\section{Introduction}

Exfoliated graphite nanoplatelets (xGnPs) are produced from exfoliating the acid intercalated graphite by rapid thermal treatments. Nanoplatelets of various sizes from $1 \mu \mathrm{m}$ to $10 \mu \mathrm{m}$ can be obtained through controlled size reduction which includes sonication and ball milling. The thickness of individual platelets is within 5-20 nm depending on the intercalation as well as the exfoliation processes. These nanoplatelets prove very effective in improving the mechanical, electrical, and barrier properties of polymer nanocomposites [1-3]. However, the enhancement in thermal conductivity is lower than expected in nanocomposites mostly due to the large thermal interface resistance between the matrix and the nanofiller [4-7].

In this paper, the thermal transport properties of $\mathrm{xGnP}$ particles were investigated and the effect of thermal interface resistance on nanoscale heat transfer was discussed. Earlier work has shown that graphene, a one-atom-thick sheet of covalently bonded carbon atoms with $\mathrm{sp}^{2}$ hybridization packed in a honeycomb crystal lattice, supports $2 \mathrm{D}$ phonon propagation that gives rise to a remarkably high thermal conductivity in the basal plane of the graphene [8]. It is difficult to use conventional techniques such as $3 \omega$, laser flash, and thermal bridge to measure thermal conductivity of this very delicate system such as single-layer graphene without causing physical damage during sample preparation and handling. Confocal micro-Raman spectroscopy, a nondestructive, optical technique has been successfully used to extract thermal properties of thin films $[9,10]$. Balandin et al. successfully measured thermal conductivity of a mechanically cleaved single layer graphene ribbon with Raman spectroscopy that uses the Raman G peak of graphene as a probe for local temperature determination at the laser spot. The measured thermal conductivity of graphene is 4400-5300 W/m K [11]. Similarly with Raman spectroscopy, Faugeras et al. used temperature readout obtained from the intensity ratio of Stokes to anti-Stokes signal to study the thermal properties of a large circular-shaped graphene membrane also obtained from micro-mechanical cleavage 
and they concluded thermal conductivity to be $600 \mathrm{~W} / \mathrm{m} \mathrm{K}$ [12]. These single layer graphenes were all suspended over a microtrench with their edges connecting to either bulk graphite or gold heat sinks. Recently, Cai et al. reported a CVD grown graphene monolayer suspended or supported over a holey $\mathrm{Au} / \mathrm{SiN}$ substrate. The thermal conductivity of graphene was measured by monitoring the graphene G peak shift with laser heating. The measured conductivities for suspended and supported graphene are $2500+1100 /-1050 \mathrm{~W} / \mathrm{m} \mathrm{K}$ and $370+650 /-320 \mathrm{~W} / \mathrm{m} \mathrm{K}$, respectively [13]. It was surmised that the huge difference is due to the phonon leakage and scattering at the substrategraphene interface. More recently, Ghosh et al. investigated the dimensional crossover of thermal transport in few-layer graphene (FLG) using the same technique, they showed that the room temperature thermal conductivity changes from $2800 \mathrm{~W} / \mathrm{m} \mathrm{K}$ to $1300 \mathrm{~W} / \mathrm{m} \mathrm{K}$ as the number of atomic planes in FLG increases from 2 to 4 [14].

Despite the numerous literature that focus on the thermal conductivity of graphene and few-layer graphene, there is no report that investigates the thermal conductivity of a monolayer film consisting of individual graphite nanoplatelets (which consists of multiple layers of graphene). Here, a monolayer film of $\mathrm{xGnP}$ was extracted from a liquid-liquid interface following the procedures reported by Biswas and Drzal [15] and deposited on a substrate for thermal conductivity measurement by confocal Raman spectroscopy that shed light on the effectiveness of those highly conductive nanoplatelets in heat conduction. In a monolayer, individual nanoplatelet is lying flat on the glass substrate and densely connected to each other resembling a percolated conductive network in a nanocomposite except that the nanoparticles are highly ordered with well-defined contacts instead of a random distribution of particles with uncontrolled contacts. Heat spreads in a $2 \mathrm{D}$ plane in the monolayer and the efficiency of heat conduction of such monolayers was studied.

\section{Experimental Details}

2.1. Materials. Exfoliated graphite nanoplatelets were prepared by exfoliating the acid intercalated natural graphite purchased from Asbury Graphite Mills, Inc, NJ. A microwave exfoliation process developed at MSU produces exfoliated material superior to conventional thermal treatments because of the fast heating rate and high energy density [2]. The volume of exfoliated graphite is over 500 times that of the initial volume occupied by graphite intercalated compounds. The surface area was measured by BrunauerEmmet-Teller (BET) using $\mathrm{N}_{2}$ adsorption at $77 \mathrm{~K}$. Two kinds of exfoliated nanoplatelets produced from different graphite sources were measured and their surface areas were determined to be $20-40 \mathrm{~m}^{2} / \mathrm{g}$ and $200-220 \mathrm{~m}^{2} / \mathrm{g}$, and are referred to as low surface area (LSA) $\mathrm{xGnP}$ and high surface area (HSA) xGnP in later discussion. Chloroform, used for liquid-liquid interface extraction of a monolayer of $\mathrm{xGnP}$ was purchased from J. T. Baker and used directly without purification.
2.2. Extraction of a Monolayer $x G n P$ at the Liquid-Liquid Interface. A monolayer of densely packed $\mathrm{xGnP}$ particles was prepared on the glass substrate following the procedure by Biswas and Drzal [15]. To summarize the process, graphite nanoplatelets were dispersed in chloroform at $0.1 \mathrm{mg} / \mathrm{mL}$. The supernatant of the suspension was obtained by $10 \mathrm{~min}$ centrifugation at $5000 \mathrm{rpm}$. Then water was added to the supernatant solution followed by high power sonication. The $\mathrm{xGnP}$ particles then quickly adsorbed at the chloroformwater interface and a monolayer was extracted on a glass slide. The samples were dried in air and then annealed at $250 \mathrm{C}$ for $2 \mathrm{~h}$. The $\mathrm{xGnP}$ coated region is $3 \mathrm{~cm} \times 2.5 \mathrm{~cm}$.

2.3. UV/Vis Spectrum. The optical transparency of the monolayer coated glass slide was measured by UV-Vis spectrometer (Perkin Elmer Lambda 900). The amount of light transmitted was measured and the light absorbed by the $\mathrm{xGnP}$ monolayer was calculated from transmission spectrum.

2.4. Scanning Electron Microscopy. A JEOL JSM-7500F scanning electron microscope was used to examine the morphology of the monolayer on the glass slide. The sample was not gold coated and the imaging was done at $5 \mathrm{kV}$ at a working distance of $4.5 \mathrm{~mm}$.

2.5. Confocal Raman Spectroscopy. A LabRAM ARAMIS laser Raman spectrometer (Horiba Scientific) with a $532 \mathrm{~nm}$ $50 \mathrm{~mW}$ DPSS laser was used to probe the $\mathrm{G}$ band of $\mathrm{xGnP}$ particles. To determine the temperature dependence of $\mathrm{G}$ peak, an INSTEC (STC 200) heating stage was used to heat the sample and a very low laser power was used to collect the Raman signal to avoid laser-induced heating. Monolayers of both LSA and HSA xGnP were prepared on the microscopic glass slides for subsequent measurements. The laser was focused on the center of the monolayer and a filter that controls different amount of laser coming through the objective lens was used to induce different levels of heating in the samples. A D1, D06, D03, D0 filter was used in each experiment that corresponds to $10 \%, 25 \%, 50 \%$, and $100 \%$ laser power. Both the x 50 and x100 objective lenses were used for each sample measured. The laser spot size $r_{0}$ was $0.24 \mu \mathrm{m}$ and $0.19 \mu \mathrm{m}$ for the $\mathrm{x} 50$ and $\mathrm{x} 100$, respectively, which were determined using $r_{0}=\lambda / \pi N A$, where $N A$ is the numerical aperture of 0.9 and 0.75 for the $\mathrm{x} 100$ and $\mathrm{x} 50$ objectives.

\section{Results and Discussions}

The formation of a stable monolayer of $\mathrm{xGnP}$ particles that were closely connected without overlapping was driven by the gain in interfacial energy that enables the nanoplatelets to be adsorbed to the oil-water interface [16-18]. Since these graphite nanosheets are highly hydrophobic on their basal planes while the oxygen functional groups on the edges render them hydrophilic, it is expected that a meniscus will be generated around the particles and "like" menisci overlap to generate large attractive capillary force to form 


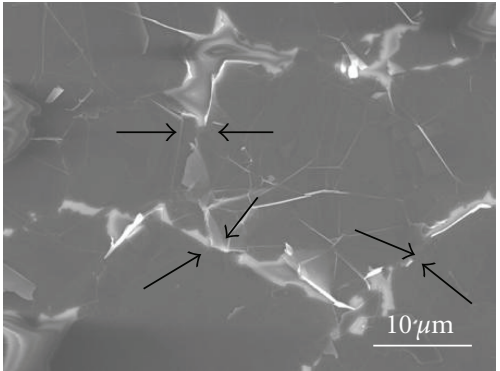

(a)

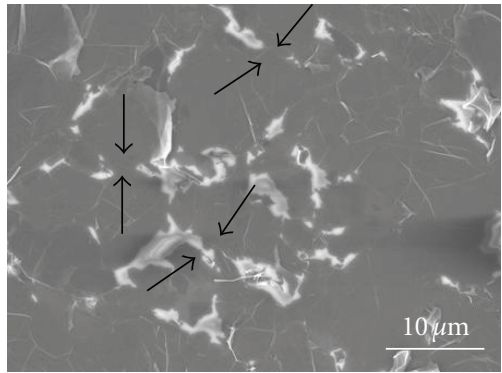

(b)

FIGURE 1: (a) SEM image of a LSA xGnP monolayer film supported on a glass substrate. (b) SEM image of a HSA xGnP monolayer film supported on a glass substrate.

a close packed structure at the liquid-liquid interface [18, 19]. While the magnitude of this lateral capillary force is negligible for spherical particles with diameter less than $10 \mu \mathrm{m}$ [20], Bowden et al. showed that, for planar shaped particles at the liquid-liquid interface, the lateral capillary force is quite significant even for particles with thicknesses of few nanometers [18]. Depending on the starting material and intercalation and exfoliation conditions, the average thickness of individual nanoplatelets may vary which will be reflected in the optical transparency measurement.

Figures 1(a) and 1(b) shows the SEM images of the two glass substrates coated with LSA and HSA xGnP monolayers. From SEM micrographs, individual nanoplatelets come into contact with each other at the edges (indicated by arrows in the figures) so well that there is no noticeable gap left between them thanks to the regular geometry of the platelet edges. In some portions of the glass substrate, gaps can be observed between nanoplatelets, but every single one is connected to the adjacent particle without overlapping for both LSA and HSA monolayers which proves the strong edge interaction among nanoplatelets. With multiple inspection of different parts of the monolayers, it is concluded that the LSA monolayer has an average particle size of around 5-10 $\mu \mathrm{m}$. In the HSA monolayer, however, more gaps exist among individual platelets and the average size of the nanoplatelet is within $1-5 \mu \mathrm{m}$. It is obvious from the SEM images that the interface density in the HSA monolayer is higher than the LSA monolayer.

\subsection{Optical Transmission and Absorption of $x G n P$ Monolayers.} The optical transparency of those monolayers was determined by scanning a blank glass slide and a $\mathrm{xGnP}$ coated slide to eliminate the effect of reflective loss from the slides. Since the reflection of the graphite nanosheets is negligibly small [21], it is not accounted for in this calculation. The amount of light absorbed was calculated by subtracting the light transmitted through the glass slide: $A$ (absorption) $=1-T$ (transmission). Figure 2 shows the percentage transmission of light for both the LSA and HSA monolayers from $350 \mathrm{~nm}$ to $1000 \mathrm{~nm}$. The optical transparency for the LSA and HSA monolayer is $20.5 \%$ and $49.6 \%$, respectively, at $532 \mathrm{~nm}$. The absorption is then calculated to be $79.5 \%$ and $50.4 \%$ for the LSA and HSA monolayers. The fact that the HSA xGnP went

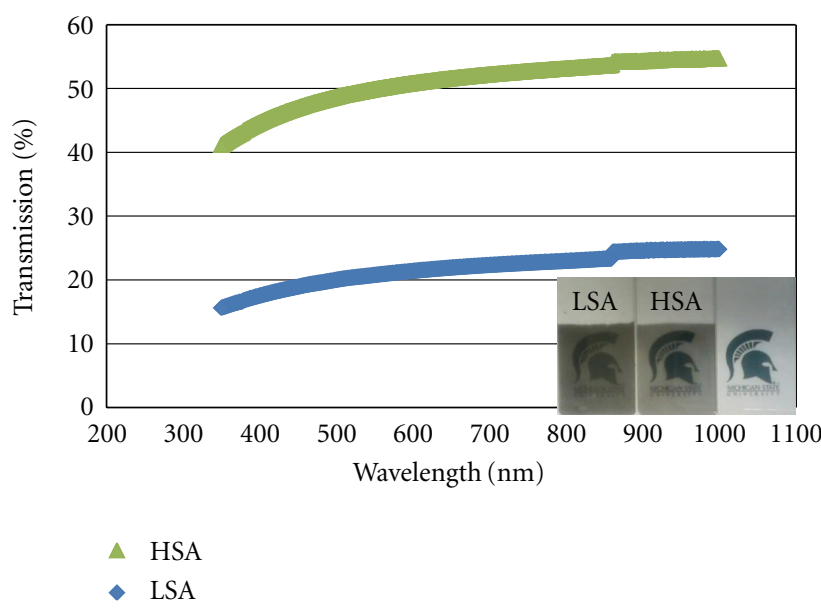

FIGURE 2: Percent transmission of light for LSA and HSA monolayers scanning from $350 \mathrm{~nm}$ to $1000 \mathrm{~nm}$. Inset is the photo of LSA and HSA coated monolayers supported on glass substrates.

through better intercalation and exfoliation contributes to the production of much thinner nanoplatelets, which boosts the optical transparency. The thickness of the individual HSA $\mathrm{xGnP}$ and LSA $\mathrm{xGnP}$ were previously measured to be around $10 \mathrm{~nm}$ and $20 \mathrm{~nm}$, respectively, by AFM [15].

3.2. Confocal Micro-Raman Spectroscopy Analysis. The laserinduced heating of the graphite nanoplatelets causes a red shift of the $G$ band because of bond softening. The $G$ band shift is believed to be linearly dependent on the temperature of the sample [22]. A heating stage that heats individual graphite nanoplatelets at a very low laser power was used to obtain a temperature calibration profile. Two measurements were taken at each temperature up to $473 \mathrm{~K}$. The slope of the temperature dependence of $G$ peak was determined to be $-0.021 \mathrm{~cm}^{-1} / \mathrm{K}$ shown in Figure 3. However, in the monolayer samples, heat absorbed by the graphite nanoplatelets propagates to the edge of the nanoplatelet and the large thermal interface resistance is expected to scatter the phonons (lattice vibrations). This is in stark contrast to constructing a graphite or Au heat sink around an individual nanoplatelets. A phonon being transported along 


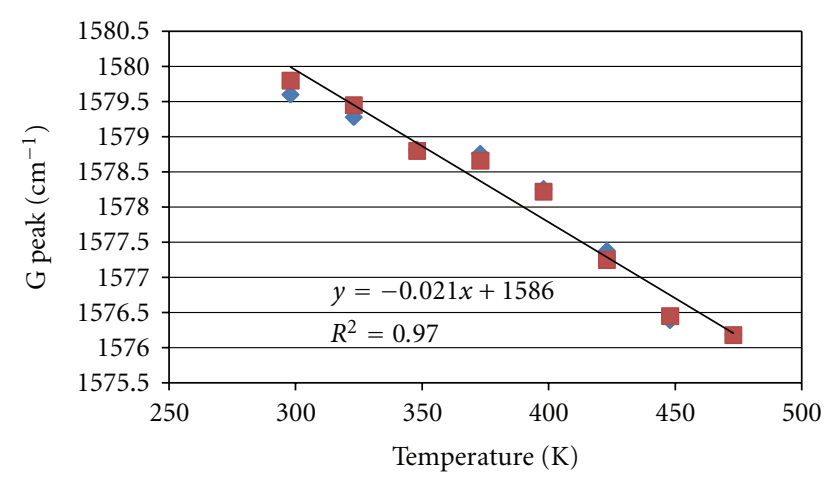

FIGURE 3: Raman G band shift of $\mathrm{xGnP}$ with temperature and linear curve fitting.

the monolayer encounters numerous interfaces that reduce the heat conduction efficiency. It is necessary to focus the laser on the center of the monolayer to ensure that heat spreads uniformly in a circular geometry. In fact, if the laser beam was focused on the edge of the sample, the local temperature will increase much higher due to the presence of a boundary that impedes heat dissipation. During the experiment, the edges of the monolayer sample was kept at ambient temperature at around $300 \mathrm{~K}$, additional heat sinks were not used because of the huge surface area of the monolayer compared with the laser spot size. The Raman G-peak shift was collected at different laser power levels (i.e., $100 \%, 50 \%, 25 \%$ and $10 \%$ ) with the selection of a proper filter. Figures 4(a) and 4(b) show the G-peak shift at different power levels for both the LSA and HSA monolayers. The presence of oxygen functional groups on the edges of $\mathrm{xGnP}$ are likely to alter the carbon bonding from $\mathrm{sp}^{2}$ to $\mathrm{sp}^{3}$ type, and this gives rise to a D band in the Raman signal $\left(1350 \mathrm{~cm}^{-1}\right)$. Due to the difference in the size of the nanoplatelets in LSA and HSA monolayer, the HSA sample has more edge areas exposed to the laser and this provides a possible explanation to a higher D band observed in HSA samples.

\subsection{Calculation of the Thermal Conductivity of $x G n P$ Mono-} layers. The temperature rise at the center of the monolayer caused by the Gaussian-shaped laser heating is proportional to the absorbed laser power and inversely proportional to the efficiency of heat conduction. The volumetric laser heating is $q_{v}=q_{0} / t \exp \left(-r^{2} / r_{0}^{2}\right)$ with $r_{0}$ being the laser spot size, $r$ being the radial position measured from the center of the laser beam. Here, $q_{0}$ is the absorbed laser power per unit area at the laser spot and $t$ is the thickness of monolayer which is the same as the thickness of an individual nanoplatelets. The glass substrate $(\kappa=1 \mathrm{~W} / \mathrm{m} \mathrm{K})$ interacts with the phonons and causes phonon leakage which reduces the thermal conductivity. The temperature distribution of the supported monolayer can be obtained by the steady state form of the heat diffusion equation in cylindrical coordinate following Cai's approach [13]:

$$
\frac{1}{r} \frac{d}{d r}\left(r \frac{d T}{d r}\right)-\frac{g}{\kappa t}\left(T-T_{a}\right)+\frac{q_{v}}{\kappa}=0
$$

where $g$ is the interfacial thermal conductance per unit area between the monolayer and the substrate as well as the air molecules. $\kappa$ is the thermal conductivity of the monolayer, $T_{a}$ is the ambient temperature. From the Gaussian-shaped laser heating, the total absorbed power by the monolayer $Q$ is

$$
Q=t \int q_{v} 2 \pi r d r=q_{0} \pi r_{0}^{2}
$$

Let $\theta=T-T_{a}$ and $z=(g / \kappa t)^{1 / 2} r$, (1) can be converted to a nonhomogeneous Bessel's equation:

$$
\frac{\partial^{2} \theta}{\partial z^{2}}+\frac{1}{z} \frac{\partial \theta}{\partial z}-\theta=-\frac{q_{0}}{g} \exp \left(-\frac{z^{2}}{z_{0}^{2}}\right)
$$

The solution to the above equation is

$$
\theta(z)=C_{1} I_{0}(z)+C_{2} K_{0}(z)+\theta_{p}(z),
$$

where $I_{0}(z)$ and $K_{0}(z)$ are the zero-order modified Bessel functions of the first and second kind. The particular solution $\theta_{p}(z)$ can be obtained using the variation of parameters method and is found to depend on $q_{0} / g$ [23]. The temperature rise in the monolayer measured by Raman laser is

$$
\theta_{m}=\frac{\int_{0}^{\infty} \theta(z) \exp \left(-z^{2} / z_{0}^{2}\right) z d z}{\int_{0}^{\infty} \exp \left(-z^{2} / z_{0}^{2}\right) z d z}
$$

The measured thermal resistance defined as $R=\theta_{m} / Q$ can be calculated from (2) and (5). Given the temperature dependence of Raman $G$ peak, the temperature rise $\theta_{m}$ and absorbed power $Q$ is found to be linearly related as is shown in Figures 5(a) and 5(b) and the slope of which yields $R_{\mathrm{LSA}}$ equal to $1.12 \times 10^{4} \mathrm{~K} / \mathrm{W}$ and $1.61 \times 10^{4} \mathrm{~K} / \mathrm{W}$, respectively, for the LSA monolayer with x50 and x100 objectives. Similarly, $R_{\mathrm{HSA}}$ equals $2.31 \times 10^{4} \mathrm{~K} / \mathrm{W}$ and $3.54 \times 10^{4} \mathrm{~K} / \mathrm{W}$ for the HSA monolayer with $\mathrm{x} 50$ and $\mathrm{x} 100$ objectives. The ratio of the two $R$ values is found to be dependent only on $g / \kappa$. With one of the $R$ values measured with either objective, $g^{2} / \kappa$ can be further obtained. After a curve fitting procedure for $g / \kappa$ and $g^{2} / \kappa$, it is found that $g \approx 50 \mathrm{MW} / \mathrm{m}^{2} \mathrm{~K}$ and $\kappa_{\mathrm{LSA}} \approx$ $380 \mathrm{~W} / \mathrm{m} \mathrm{K}$ for the LSA monolayer while $g \approx 30 \mathrm{MW} / \mathrm{m}^{2} \mathrm{~K}$ and $\kappa_{\mathrm{HSA}} \approx 290 \mathrm{~W} / \mathrm{m} \mathrm{K}$ for the HSA monolayer. Due to the difference in $\mathrm{xGnP}$ size, the HSA monolayer has a higher interface density than the LSA monolayer which might cause the reduction in overall efficiency of heat conduction due to strong phonon scattering at the particle interfaces.

\section{Conclusions}

A densely packed monolayer of $\mathrm{xGnP}$ was successfully extracted from a chloroform-water interface driven by interfacial energy minimization and attractive capillary interactions. Confocal micro-Raman spectroscopy was used as a heater and a thermometer to probe the heat conduction 

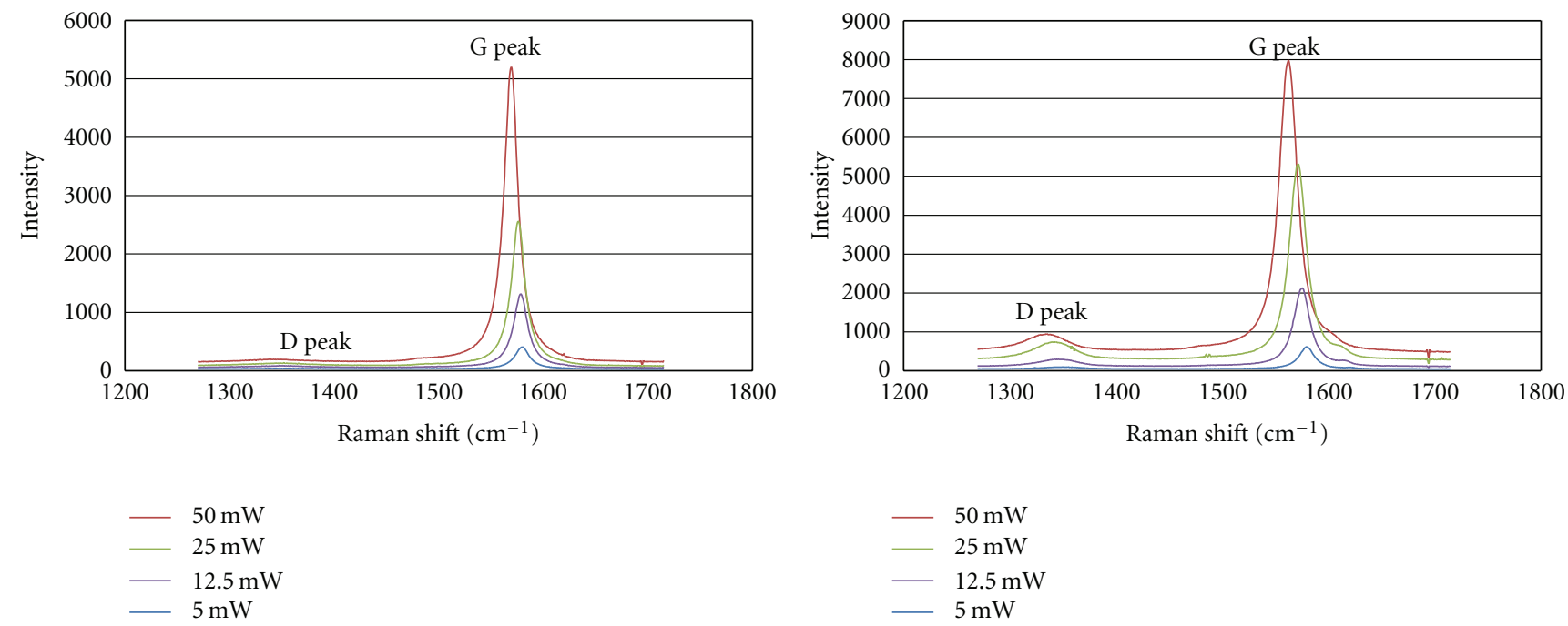

(a)

(b)

FIGURE 4: (a) Raman G peak shift of the LSA monolayer at different laser power levels. (b) Raman G peak shift of the HSA monolayer using different laser power levels.

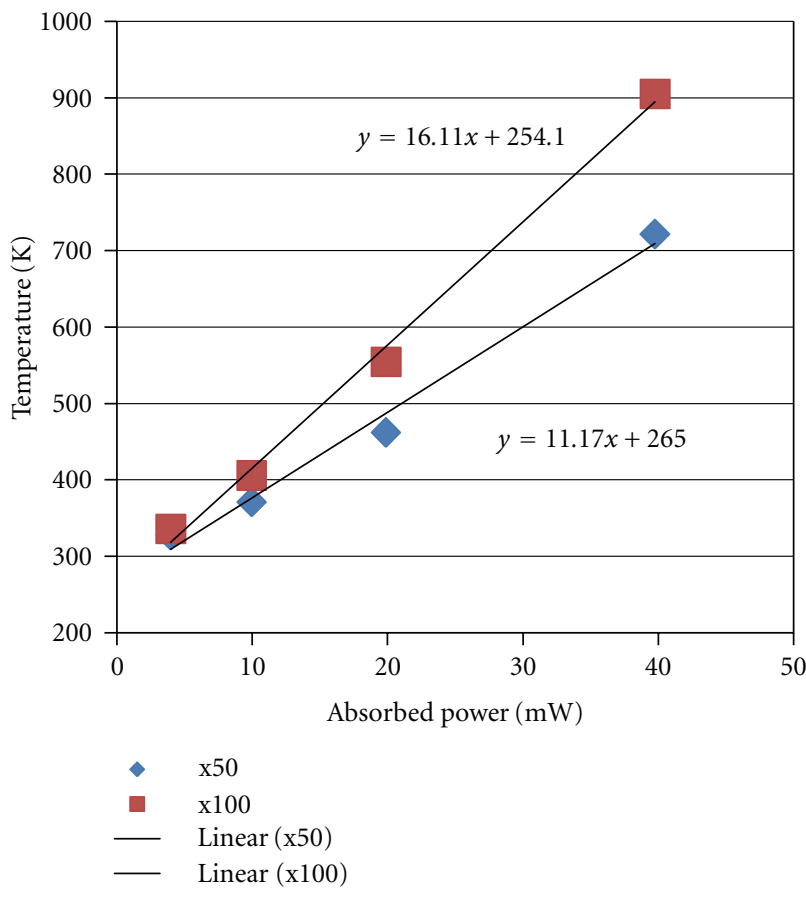

(a)

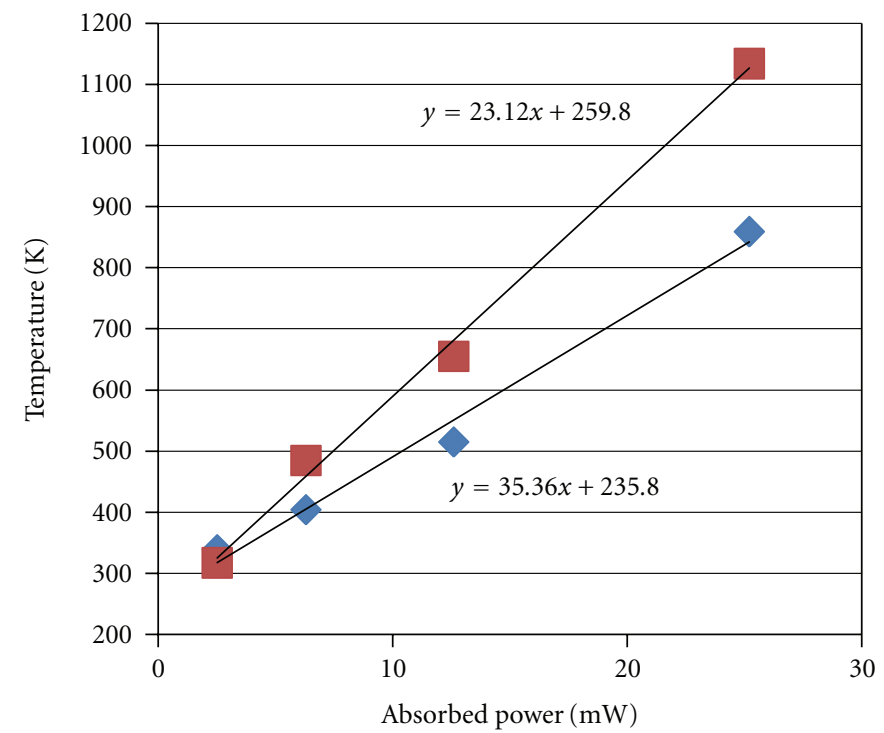

$\begin{array}{ll}-\quad & \text { x50 } \\ -\quad & \text { x100 } \\ -\quad \text { Linear }(x 50) \\ -\quad \text { Linear }(x 100)\end{array}$

FIGURE 5: (a) Local temperature of an individual xGnP at the center of a LSA monolayer versus the absorbed laser power with x50 and x100 objectives. The solid lines represent the linear curve fittings. (b) Local temperature of an individual xGnP at the center of a HSA monolayer versus the absorbed laser power with x50 and x100 objectives. The solid lines represent the linear curve fittings.

efficiency of monolayers made from $\mathrm{xGnP}$ of different sizes and thicknesses. The presence of interfaces in the monolayers significantly reduces the thermal conductivity in this assembly of graphene nanoplatelets by almost an order of magnitude lower than the highest reported value for an individual nanoplatelets measured by the same technique. However, the in-plane thermal conductivities of both monolayers are still high with negligible cross plane conduction making it a promising candidate for applications that require fast $2 \mathrm{D}$ heat dissipation. 


\section{Acknowledgment}

The authors wish to acknowledge the support of the State of Michigan 21st Century Jobs Fund for partial support of this research.

\section{References}

[1] S. Stankovich, D. A. Dikin, G. H. B. Dommett et al., "Graphene-based composite materials," Nature, vol. 442, no. 7100, pp. 282-286, 2006.

[2] H. Fukushima, Graphite reinforcements in polymer nanocomposites, Ph.D. thesis, Department of Chemical Engineering and Materials Science, Michigan State University, East Lansing, Mich, USA, 2003.

[3] K. Kalaitzidou, H. Fukushima, and L. T. Drzal, "Multifunctional polypropylene composites produced by incorporation of exfoliated graphite nanoplatelets," Carbon, vol. 45, no. 7, pp. 1446-1452, 2007.

[4] M. T. Hung, O. Choi, Y. S. Ju, and H. T. Hahn, "Heat conduction in graphite-nanoplatelet-reinforced polymer nanocomposites," Applied Physics Letters, vol. 89, no. 2, Article ID 023117, 2006.

[5] C. W. Nan, G. Liu, Y. Lin, and M. Li, "Interface effect on thermal conductivity of carbon nanotube composites," Applied Physics Letters, vol. 85, no. 16, pp. 3549-3551, 2004.

[6] S. T. Huxtable, D. G. Cahill, S. Shenogin et al., "Interfacial heat flow in carbon nanotube suspensions," Nature Materials, vol. 2, no. 11, pp. 731-734, 2003.

[7] N. Shenogina, S. Shenogin, L. Xue, and P. Keblinski, "On the lack of thermal percolation in carbon nanotube composites," Applied Physics Letters, vol. 87, no. 13, Article ID 133106, 3 pages, 2005.

[8] D. L. Nika, S. Ghosh, E. P. Pokatilov, and A. A. Balandin, "Lattice thermal conductivity of graphene flakes: comparison with bulk graphite," Applied Physics Letters, vol. 94, no. 20, Article ID 203103, 2009.

[9] S. Périchon, V. Lysenko, B. Remaki, D. Barbier, and B. Champagnon, "Measurement of porous silicon thermal conductivity by micro-Raman scattering," Journal of Applied Physics, vol. 86, no. 8, pp. 4700-4702, 1999.

[10] V. Lysenko, S. Perichon, B. Remaki, D. Barbier, and B. Champagnon, "Thermal conductivity of thick meso-porous silicon layers by micro-Raman scattering," Journal of Applied Physics, vol. 86, no. 12, pp. 6841-6846, 1999.

[11] A. A. Balandin, S. Ghosh, W. Bao et al., "Superior thermal conductivity of single-layer graphene," Nano Letters, vol. 8, no. 3, pp. 902-907, 2008.

[12] C. Faugeras, B. Faugeras, M. Orlita, M. Potemski, R. R. Nair, and A. K. Geim, "Thermal conductivity of graphene in corbino membrane geometry," ACS Nano, vol. 4, no. 4, pp. 1889-1892, 2010.

[13] W. Cai, A. L. Moore, Y. Zhu et al., "Thermal transport in suspended and supported monolayer graphene grown by chemical vapor deposition," Nano Letters, vol. 10, no. 5, pp. 1645-1651, 2010.

[14] S. Ghosh, W. Bao, D. L. Nika et al., "Dimensional crossover of thermal transport in few-layer graphene," Nature Materials, vol. 9, no. 7, pp. 555-558, 2010.

[15] S. Biswas and L. T. Drzal, "A novel approach to create a highly ordered monolayer film of graphene nanosheets at the liquidliquid interface," Nano Letters, vol. 9, no. 1, pp. 167-172, 2009.
[16] Y. Lin, H. Skaff, T. Emrick, A. D. Dinsmore, and T. P. Russell, "Nanoparticle assembly and transport at liquid-liquid interfaces," Science, vol. 299, no. 5604, pp. 226-229, 2003.

[17] W. H. Binder, "Supramolecular assembly of nanoparticles at liquid-liquid interfaces," Angewandte Chemie-International Edition, vol. 44, no. 33, pp. 5172-5175, 2005.

[18] N. Bowden, A. Terfort, J. Carbeck, and G. M. Whitesides, "Assembly of mesoscale objects into ordered two-dimensional arrays," Science, vol. 276, no. 5310, pp. 233-235, 1997.

[19] N. Bowden, S. R. J. Oliver, and G. M. Whitesides, "Mesoscale self-assembly: capillary bonds and negative menisci," Journal of Physical Chemistry B, vol. 104, no. 12, pp. 2714-2724, 2000.

[20] P. A. Kralchevsky, V. N. Paunov, N. D. Denkov, I. B. Ivanov, and K. Nagayama, "Energetical and force approaches to the capillary interactions between particles attached to a liquidfluid interface," Journal of Colloid And Interface Science, vol. 155, no. 2, pp. 420-437, 1993.

[21] R. R. Nair, P. Blake, A. N. Grigorenko et al., "Fine structure constant defines visual transparency of graphene," Science, vol. 320, no. 5881, p. 1308, 2008.

[22] I. Calizo, A. A. Balandin, W. Bao, F. Miao, and C. N. Lau, "Temperature dependence of the raman spectra of graphene and graphene multilayers," Nano Letters, vol. 7, no. 9, pp. 2645-2649, 2007.

[23] M. Greenberg, Advanced Engineering Mathematics, Prentice Hall, Upper Saddle River, NJ, USA, 2nd edition, 1998. 

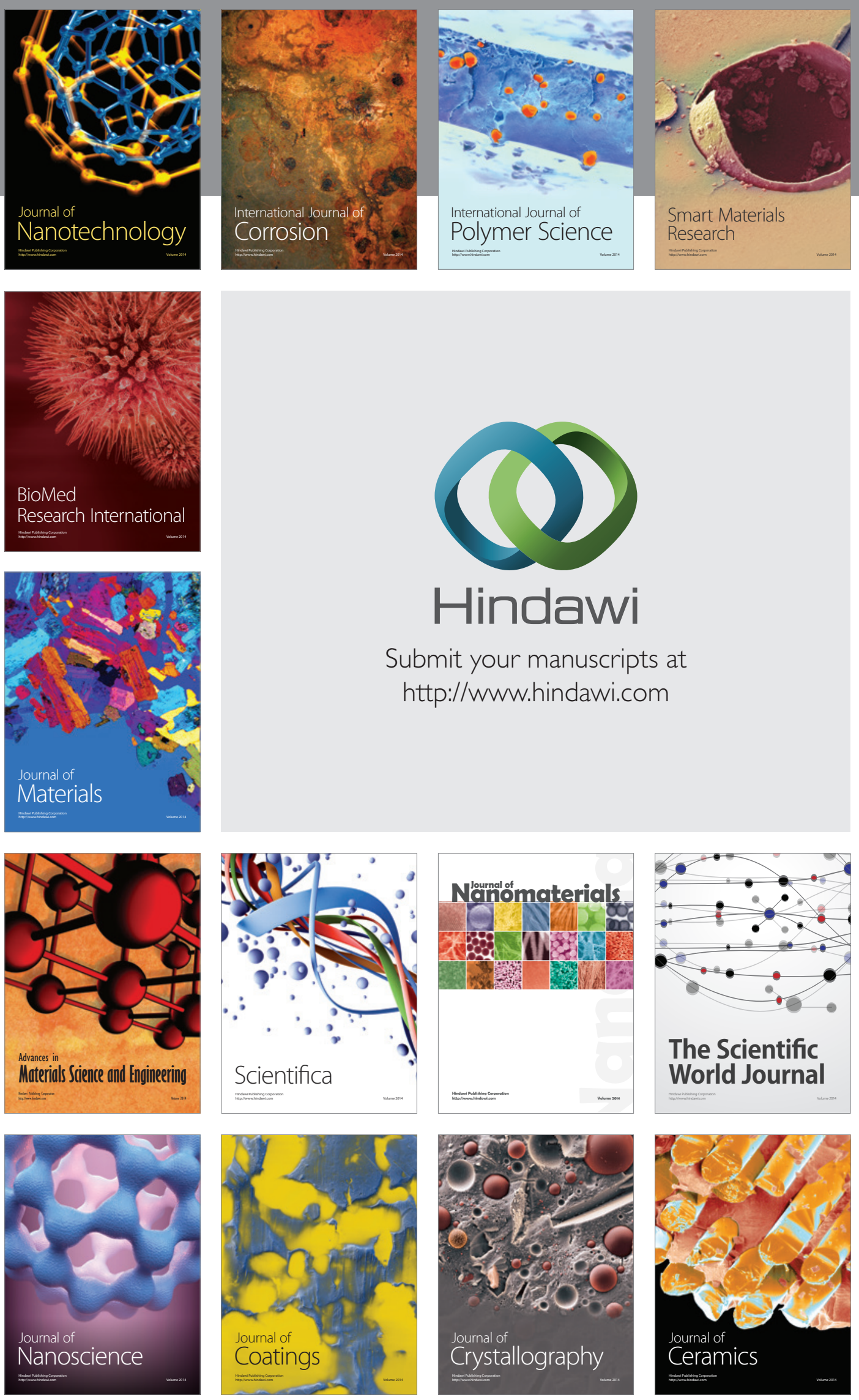

The Scientific World Journal

Submit your manuscripts at

http://www.hindawi.com

\section{World Journal}

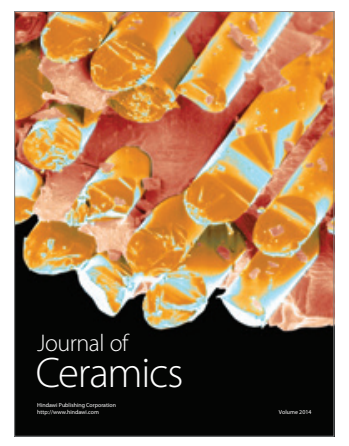

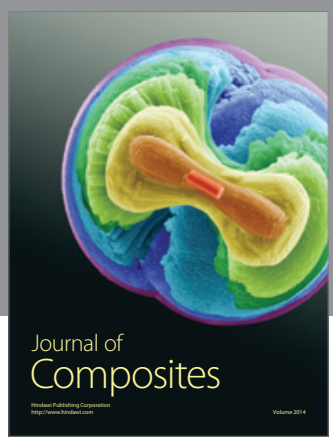
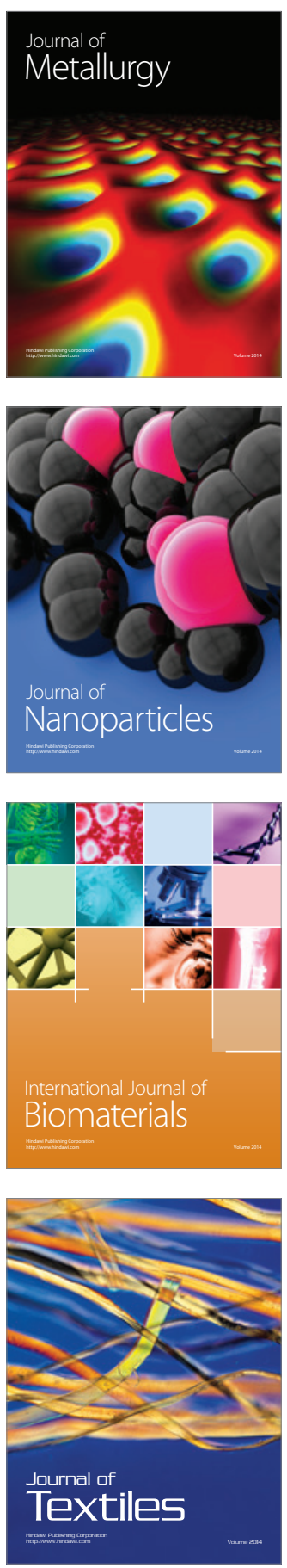